Check for updates

Cite this: RSC Adv., 2018, 8, 12315

Received 22nd January 2018

Accepted 19th March 2018

DOI: 10.1039/c8ra00632f

rsc.li/rsc-advances

\section{Microencapsulated sunblock nanoparticles based on zeolitic imidazole frameworks for safe and effective UV protection}

\author{
Lixian $\mathrm{Xu}{ }^{\mathrm{a}} \mathrm{Di}$ Wu,${ }^{a}$ Bingrong Zhou, ${ }^{\mathrm{a}}$ Yang $\mathrm{Xu},{ }^{\mathrm{a}}$ Wenjuan Wang, ${ }^{\mathrm{b}}$ Dinghua Yu (D) *b \\ and Dan Luo*a
}

Sunscreen is believed to protect human skin from photo damage due to UV exposure. However, substantial concerns remain associated with skin contact with UV filters and the subsequent reactive oxygen species from photoactivation of UV filters. Herein, we show that the microencapsulation of octyl $p$ methoxycinnamate (OMC), a typical UV filter, into the nanoporous structure of zeolitic imidazole frameworks (ZIF-8) prevents the skin exposure to UV filters while improving UV protection performance. Meanwhile, the UV filter photostability has been obviously improved due to the nanoconfinement effect. Microencapsulated OMC in ZIF-8 adhered to the stratum corneum could not only reduce OMC transdermal penetration, but also prevent skin exposure to the deleterious reactive oxygen species (ROS) from photoactivation of OMC. Therefore, this microencapsulated UV filter system based on a metal organic frameworks microporous polymer would find important applications in skin care products through providing safe and effective UV protection.

\section{Introduction}

Sunlight is very important to all living organisms on earth because it can provide sufficient energy for life activities. For human health, harmful ultraviolet (UV) radiation from sunlight could induce a series of deleterious effects on skin such as erythema formation, immunosuppression, oxidative stress, photoaging, DNA and RNA damage and skin cancers. ${ }^{1-3}$ In order to avoid UV radiation damage, UV filters have been developed as effective materials and have been widely employed in personal care products such as lotions, sunscreens and cosmetic products. $^{4-6}$ These UV filters, including inorganic and organic components, could protect human skin from sunburn, photoaging and skin cancer through reducing UV absorption of human skin through different mechanisms. Inorganic sunscreens reduce UV damage through reflection, scattering and diffraction of the UV radiation, whereas organic sunscreens reduce UV damage through absorption.

Inorganic UV filters, such as $\mathrm{TiO}_{2}$, are insoluble mineralbased nanoparticles, which would not penetrate into the skin and reduce the irritation risk to sensitive skin. However, the anatase-type $\mathrm{TiO}_{2}$ is a kind of photocatalytic material. In the presence of oxygen, water and light, the photoexcited $\mathrm{TiO}_{2}$ could produce hydroxyl and peroxide radicals, and successively

${ }^{a}$ Department of Dermatology, The First Affiliated Hospital of Nanjing Medica University, Nanjing, Jiangsu, P. R. China.E-mail: daniluo2005@163.com

${ }^{b}$ College of Biotechnology and Pharmaceutical Engineering, Nanjing Tech University, Nanjing, Jiangsu, P. R. China. E-mail: yudh@njtech.edu.cn produce reactive oxygen species (ROS) in the skin, which could induce cyto- and genotoxicity through deactivating enzymes and decomposing biomolecules such as RNA and membrane proteins. $^{7-9}$ Therefore, many researches have been devoted to improve the safety of inorganic UV filter. For instance, $\mathrm{ZnO}$ and $\mathrm{TiO}_{2}$ has been encapsulated by biologically inert $\mathrm{SiO}_{2}$ (ref. 10) or cellulose nanocrystals, ${ }^{\mathbf{1 1}}$ and the core/shell structure has lowered the risk of DNA damage without changing their optoelectronic properties.

In comparison, organic UV filters could absorb UV spectrum as molecule state, and many synthetic organic matter and natural product extract could be ascribed into this category. trans-Octyl methoxycinnamate (OMC) is the most commonly used UVB filter and is approved for use in many personal care products such as sunscreens, lip balms and other protection products. ${ }^{12}$ However, many researches have demonstrated that OMC could penetrate into the skin, and could be detected in urine, human blood plasma and breast milk of women after topical application. ${ }^{13-16}$ Moreover, many researches ${ }^{17-19}$ have demonstrated that $\mathrm{OMC}$ could be an endocrine disruptor, which showed estrogenic activity both in vivo (rats) and in vitro (MCF-7 breast cancer cells) and caused a marked decrease in murine thyroxine levels. In addition, the topical application of OMC has produced some side effects such as skin irritation and allergic dermatitis. Moreover, OMC could be subject to photodegradation, and produce many potentially hazardous photoproducts such as reactive oxygen species (ROS), which have been demonstrated to be more toxic to mammalian cells than OMC itself. ${ }^{20}$ In order to minimize skin contact with organic 
filters and the systemic absorption of UV filters penetrating the skin, many organic UV filters encapsulation technologies ${ }^{\mathbf{2 1 - 2 5}}$ have been developed. Saltzman et $a .^{21}$ reported that the encapsulation of the organic UV filters in polymer nanoparticles, which could adhere to the skin well, do not penetrate into the skin and blood stream, and reactive oxygen species do not generate in cellular structure. Comparing with these bioadhesive nanoparticles, metal-organic frameworks (MOFs) could provide more versatile application due to their unique structural characteristics. ${ }^{26}$

Zeolitic imidazole frameworks (ZIFs) are a kind of metalorganic frameworks (MOFs) synthesized from metal ions and imidazole liners. ${ }^{27,28}$ The synthesis of ZIF-8 or MAF-4 has been reported $^{29,30}$ and recently has been used to encapsulate pyrene for optical sensing of molecular oxygen. ${ }^{31}$ Recently, ZIF-8 or MAF-4 has been used to prepare optical ceramics, which would find applications related with adsorption, separation, sensing, etc. $^{32}$ The typical characteristics of ZIFs including structural diversity, high surface area, tunable pore sizes, biocompatibility and functionalities, have made them promising candidates for biomedical applications. ${ }^{33-37}$ For example, modification of the imidazole liner in ZIFs has been used for nucleic acid detection $^{34}$ and intracellular imaging of hydrogen sulfide. ${ }^{35}$ Meanwhile, the higher aqueous stability and large pore volume of ZIFs have made them nanocarrier for drug delivery. ${ }^{36,37}$

Herein, we report for the first time that nanoscale ZIF-8, selfassembled from $\mathrm{Zn}^{2+}$ and 2-methylimidazole (Fig. 1), could encapsulate OMC to minimize UV filters contact with skin. The structure and UV protective performance of OMC/ZIF-8 hybrid nanoparticles have been characterized by a series of physicochemical protocols including XRD, SEM, FT-IR, UV-Vis, and $\mathrm{N}_{2}$ sorption. The UV protective performance and photostability of OMC/ZIF-8 have been studied through UV-Vis spectrometer. Reactive oxygen species (ROS) from OMC photo activation has been characterized by fluorescence method with dihydrorhodamine (DHR) as probe molecules. The transdermal penetration of free OMC and microencapsulated OMC/ZIF- 8 has been compared through Franz diffusion cell. The results showed that the microencapsulated OMC/ZIF-8 hybrid nanoparticles had improved UV protective performance and photostability, lowered release ratio, and avoided skin exposure to UV filter and the subsequent reactive oxygen species.

\section{Experimental}

\section{Materials}

2-Methylimidazole, zinc nitrate hexahydrate, sodium chloride, lactic acid, urea, ammonia and trans-octyl methoxycinnamate were purchased from Aladdin reagents (shanghai) co., ltd. Dihydrorhodamine (DHR) was purchased from Sigma-Aldrich Co. Other reagents are all of analytical grade. All chemicals were used as received without further purification.

\section{Synthesis procedure}

Synthesis of ZIF-8 nanocrystal materials. Blank ZIF-8 and microencapsulated OMC/ZIF-8 materials have been prepared

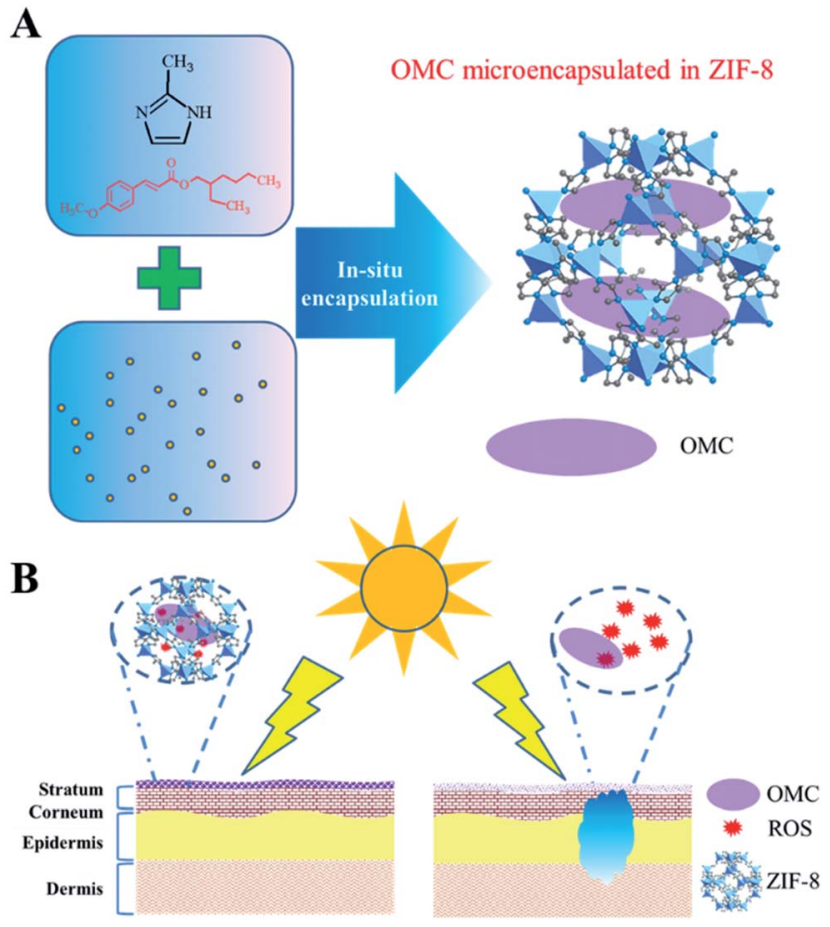

Fig. 1 Microencapsulated OMC in zeolitic imidazole frameworks for safe sunblock agent. (A) In situ micro-encapsulation diagram of OMC in ZIF- 8 materials. Since the OMC molecular size is larger than the pore size of ZIF-8, the encapsulation could only be realized through the in situ process. (B) After sunlight exposure, free OMC produces deleterious $R O S$ that could penetrate into skin tissue, and damages adjacent tissue. Microencapsulated OMC in ZIF-8 could confine these deleterious ROS species within the pore structure, and prevent ROS-mediated toxicity.

according to the method reported by Michael Wiebcke et al. ${ }^{38}$ with minor modification. Typically, $200 \mathrm{~mL}$ of methanol solution of zinc nitrate hexahydrate $\left(50 \mathrm{mmol} \mathrm{L}^{-1}, 2.97 \mathrm{~g}, 200 \mathrm{~mL}\right)$ is rapidly poured into $200 \mathrm{~mL}$ of methanol solution of 2-methylimidazole (400 mmol L ${ }^{-1}, 6.57 \mathrm{~g}, 200 \mathrm{~mL}$ ) under vigorously stirring with magnetic bar. The mixed solution is stirred for $8 \mathrm{~h}$ at room temperature, and the transparent solution turns slowly turbid. After reaction, the nanocrystals are separated from the milky dispersion by centrifugation, and the solid section was washed with fresh methanol. And the as-synthesized ZIF-8 products are dried for $8 \mathrm{~h}$ at $40{ }^{\circ} \mathrm{C}$.

Microencapsulated OMC/ZIF-8 hybrid materials. OMC/ZIF-8 hybrid materials were prepared through in situ microencapsulation protocol. In a typical process, the $200 \mathrm{~mL}$ methanol solution of 2-methylimidazole of $400 \mathrm{mmol} \mathrm{L}^{-1}$ is prepared, and $0.58 \mathrm{~g} \mathrm{OMC}(2 \mathrm{mmol})$ is added to the above solution and mixed to acquire homogeneous solution. Then under stirred vigorously, $200 \mathrm{~mL}$ of methanol solution of zinc nitrate hexahydrate $\left(50 \mathrm{mmol} \mathrm{L}^{-1}, 2.97 \mathrm{~g}, 200 \mathrm{~mL}\right.$ ) was rapidly poured into the above mixed solution. The acquired mixed solution was stirred for $8 \mathrm{~h}$ at room temperature. After reaction, the hybrid OMC/ZIF-8 nanoparticles were separated from the milky dispersion by centrifugation, and the solid products were washed with fresh methanol. And the acquired OMC/ZIF-8 solid products are dried for $8 \mathrm{~h}$ at $40{ }^{\circ} \mathrm{C}$. The actual productivity of yield was about 
$74.3 \%$. In order to calculate the encapsulation efficiency of OMC, the OMC/ZIF-8 samples were dissolved in hydrochloric acid, and the OMC concentration was determined by HPLC method. The encapsulation efficiency is about $86 \%$, and the actual content of OMC in OMC/ZIF-8 is about $0.108 \mathrm{~g} \mathrm{~g}^{-1}$.

\section{Methods}

Structure characterization. The morphology evaluations of blank ZIF-8 and OMC/ZIF-8 samples were recorded using scanning electron microscopy (SEM) (SU8010, Hitachi, Japan). Before experiments, the sample was adhered to the substrate and an ultrathin coating of electrically-conducting material was deposited on the sample surface.

X-ray diffraction analysis was performed on a Philips X'Pro Xray diffractometer using $\mathrm{Cu} \mathrm{K} \alpha$ radiation. The $\mathrm{K} \alpha 1$ wavelength was $1.5406 \AA$. The X-ray source was operated at $40 \mathrm{kV}$ and $40 \mathrm{~mA}$. The diffraction data were collected at $25{ }^{\circ} \mathrm{C}$ in a $2 \theta$ range $5-60^{\circ}$ with a scanning speed of $5^{\circ} \mathrm{min}^{-1}$.

$\mathrm{N}_{2}$ sorption measurements were carried out on an ASAP 2020 instrument from Micromeritics, USA. Before sorption measurements, the samples were degassed at $423 \mathrm{~K}$ in a vacuum for $4 \mathrm{~h}$. The specific surface area and micropore volume were calculated by Brunauer-Emmet-Teller (BET) method and $t$-plot method, respectively. The pore width distribution were obtained through BJK methods.

Fourier transform infrared spectroscopy (FT-IR) was performed on an Avatar 360 FT-IR spectrometer from Nicolet using DTGS KBr as detector. The sample was mixed with $\mathrm{KBr}$ powder and tableted into a thin round piece. The absorption spectra were recorded from $4000-400 \mathrm{~cm}^{-1}$.

The OMC release from microencapsulated OMC/ZIF-8 materials was performed according to the modified method reported by Saltzman et al. ${ }^{21}$ In the typical experiments, $10 \mathrm{mg}$ OMC/ZIF-8 was placed in a dialysis tube (8000 M.W. cutoff) and dialysed against $50 \mathrm{~mL}$ of artificial sweat at $32{ }^{\circ} \mathrm{C}$ and $37{ }^{\circ} \mathrm{C}$. The artificial sweat was formulated according to EN 1811 (0.5\% $\mathrm{NaCl}, 0.1 \%$ urea, $0.1 \%$ lactic acid and the $\mathrm{pH}$ adjusted to 6.6 with $\mathrm{NH}_{4} \mathrm{OH}$ ). At each time point, $200 \mu \mathrm{L}$ solution was extracted and $200 \mu \mathrm{L}$ of fresh solution was added. The amount of OMC released was determined by UV absorption at $310 \mathrm{~nm}$ with a UVVis spectrometer.

The UV absorption efficiency of OMC/ZIF-8 was scanned through the UV absorption spectra from 250-400 nm with a UVVis spectrometer. The OMC/ZIF-8 samples were dispersed in $\mathrm{ddH}_{2} \mathrm{O}$ with OMC concentration at $0.01 \mathrm{mg} \mathrm{mL}^{-1}$. Meanwhile, free OMC dissolved in water, free OMC dissolved in ethanol, OMC dissolved in oil (OMC concentration at $0.01 \mathrm{mg} \mathrm{mL}^{-1}$ ). Blank ZIF-8, ethanol, water and oil was used as the background controls.

For quantifying the ROS amounts of free OMC and microencapsulated OMC/ZIF-8, a fluorescence method was selected with DHR as probe molecules. Free OMC and OMC/ZIF-8 at an OMC concentration of $0.1 \mathrm{mg} \mathrm{mL^{-1 }}$ were incubated with DHR in PBS buffer in quartz cuvette. Meanwhile, blank ZIF-8 and PBS control were also compared. After exposing to UVB (280-320 $\mathrm{nm}$ ), the fluorescence intensity was read using a Fluoromax-3 fluorometer (HORIBA Jobin Yvon) at excitation/emission 500/ $536 \mathrm{~nm}$.

The photostability of free $\mathrm{OMC}$ and microencapsulated OMC/ZIF-8 was measured by the UV-Vis spectrometer. The samples were placed into a quartz cuvette sealed with a Teflon stopper, then were subjected to irradiation by a $75 \mathrm{~W}$ xenon lamp at a distance of $20 \mathrm{~cm}$ (a flux of $3 \mathrm{~mW} \mathrm{~cm}^{-2}$ between 300 and $400 \mathrm{~nm}$ ). The absorption spectra were recorded hourly for four hours. The total UV dose was thus $432 \mathrm{~kJ} \mathrm{~m}^{-2}$, equivalent to 2.5 hours of summer sunlight at noon.

Transdermal penetration test. Samples of adult human skin (mean age $36 \pm 8$ years) were obtained from plastic surgery patients with written informed consent at the First Affiliated Hospital of Nanjing Medical University, which were used as in vitro model for this study. This study was approved by the Ethics Committee of the First Affiliated Hospital of Nanjing Medical University, Nanjing, China. The human skin was cut with scissors and washed by dd $\mathrm{H}_{2} \mathrm{O}$. Fats from skin tissue were removed carefully and the acquired skin was then punched into a diameter of $1.0 \mathrm{~cm}$. The treated skin sample was stored in a phosphate buffered saline (PBS) at $4{ }^{\circ} \mathrm{C}$. Before experiments, the skin was pre-equilibrated in PBS at $25{ }^{\circ} \mathrm{C}$. Then the skin specimens were placed between the two halves of the Franz diffusion cell (Huanghai, model RYJ-6A, China), and the stratum corneum side was placed facing the donor compartment.

The receiver compartment $(10 \mathrm{~mL})$ was filled with $50 \%$ ethanol in PBS solution, and $100 \mu \mathrm{L}$ receptor solution was withdrawn and the fresh solution was supplemented at the given intervals. The whole transdermal penetration test was performed under constant stirring at $35 \pm 1{ }^{\circ} \mathrm{C}$. The $\mathrm{OMC}$ concentration was determined by a high-performance liquid chromatography (Diamond, Ultimate 3000, USA) method with a C18 reverse phase column (Biorad) and the UV detector at $310 \mathrm{~nm}$. The mobile phase was composed of acetonitrile and water $(95: 5, \mathrm{v} / \mathrm{v})$ at a flow rate of $1 \mathrm{~mL} \mathrm{~min}^{-1}$. The OMC quantification was performed according to internal standard method based on the calibration curves. The model of in vitro transdermal diffusion is based on the Fick's first law of diffusion and is showed as the following equation.

$$
J=-D \frac{\mathrm{d} c}{\mathrm{~d} x}
$$

where $J$ is the mass flux; $D$ is the diffusion coefficient; $\mathrm{d} c$ is the difference of concentration; $\mathrm{d} x$ is the distance of flow path. The lag time was calculated according to the $x$-intercept derived from the linear regression line.

\section{Results and discussion}

\section{Morphology}

The blank ZIF-8 and microencapsulated OMC/ZIF-8 have been characterized by scanning electron microscopy (SEM), and the corresponding results have been shown in Fig. 2. From Fig. 2, blank ZIF-8 and microencapsulated OMC/ZIF-8 samples show the similar nanoparticles morphology, and the size is about 50$60 \mathrm{~nm}$, which indicates that microencapsulation of OMC in ZIF8 has no obvious influence on ZIF-8 morphology. From Fig. 2(A) 


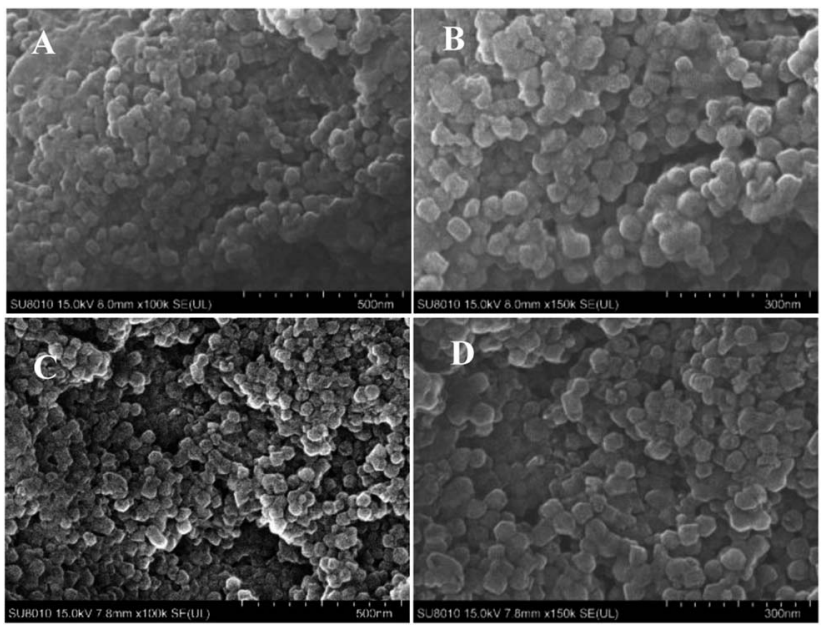

Fig. 2 The morphology results of blank ZIF-8 and OMC/ZIF-8 disclosed by scanning electron microscopy techniques. $(A)$ and $(B)$ : blank ZIF-8; (C) and (D): microencapsulated OMC/ZIF-8.

and (B), blank ZIF-8 samples show the adhesion of nanoparticles. Different with the regular polyhedron morphology of ZIF-8 materials reported in the literatures, the morphology of these as-synthesized ZIF-8 and OMC/ZIF-8 samples is close to sphere object, which could be induced by the insufficient crystallization process. As shown in Fig. 2(C) and (D), microencapsulated OMC/ZIF-8 materials consist of isolated polyhedron nanoparticles, moreover, some inter-particles space has formed as shown in Fig. 2(D), which indicated that OMC could affect the ZIF-8 crystallization process through hydrophobic interactions.

\section{Structures}

Powder X-ray diffraction (PXRD) was used to study the crystal structure of blank ZIF-8 and OMC/ZIF-8, and the diffraction patterns have been shown in Fig. 3(A). Blank ZIF-8 materials showed several diffraction peaks at $7.31^{\circ}, 10.45^{\circ}, 12.73^{\circ}, 14.70^{\circ}$, $16.46^{\circ}$ and $18^{\circ}$, which could be attributed to (011), (002), (112), (022), (013) and (222), respectively. ${ }^{38}$ Because OMC is a kind of amorphous compound, which would not show the typical X-ray diffraction peaks. Meanwhile, OMC/ZIF-8 showed the similar diffraction patterns to the blank ZIF-8 sample, which indicates that OMC don't influence the crystal structure of ZIF-8.

Fig. 3(B) showed the Fourier transform infrared spectra of blank ZIF-8 and microencapsulated OMC/ZIF-8 materials. The strong absorption between 1350 and $1500 \mathrm{~cm}^{-1}$ could be attributed to the imidazole ring stretching vibration. The absorption peak at $1586 \mathrm{~cm}^{-1}$ could be assigned as the $\mathrm{C}=\mathrm{N}$ stretching mode. The $\mathrm{N}-\mathrm{Zn}$ stretching vibration could be observed at $421 \mathrm{~cm}^{-1} \cdot{ }^{39}$ Comparing with blank ZIF-8 material, OMC/ZIF-8 samples showed several new weak absorption peaks. The absorption at $1254 \mathrm{~cm}^{-1}$ could be assigned to the stretching vibration of $\mathrm{C}-\mathrm{O}$ of ester bonds, and the absorption peak at $1732 \mathrm{~cm}^{-1}$ could be attributed to the stretching vibration of $\mathrm{C}=\mathrm{O}$ bonds. These new absorption peaks were induced by OMC molecules, which indicated that OMC had been encapsulated into the ZIF-8 porous structures.
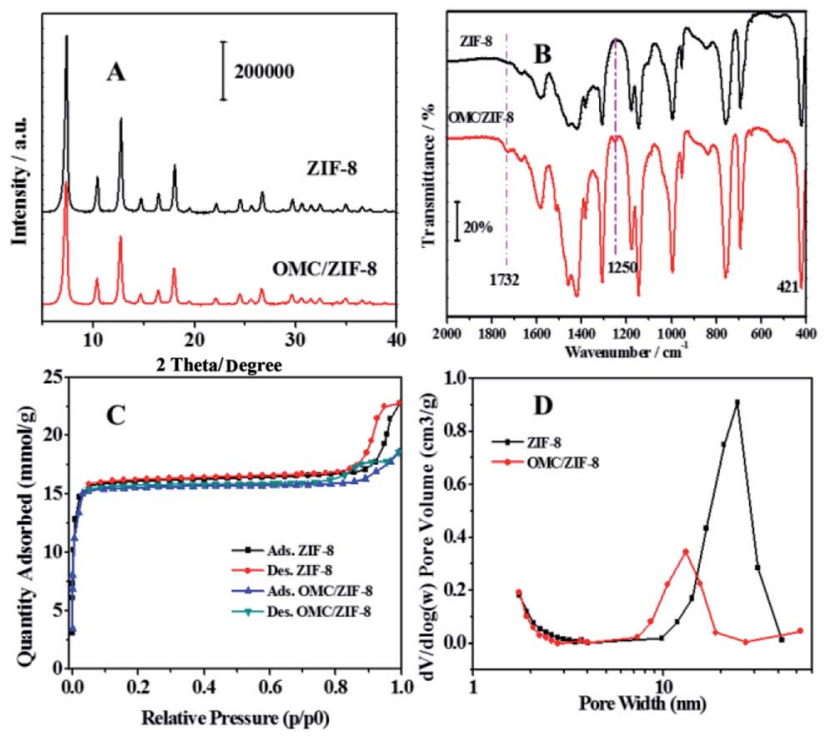

Fig. 3 The structure characterization of blank ZIF-8 and microencapsulated OMC/ZIF-8. (A) Powder X-ray diffraction patterns; (B) Fourier transform infrared spectra; (C) $\mathrm{N}_{2}$ adsorption-desorption isotherms at $77 \mathrm{~K}$; (D) pore size distribution curves.

As shown in Fig. 3(C), both the blank ZIF-8 and OMC/ZIF-8 showed the typical type I adsorption isotherms according to IUPAC recommendation, which indicated that the assynthesized blank ZIF-8 and OMC/ZIF-8 samples were a kind of microporous materials. Meanwhile, both $\mathrm{N}_{2}$ sorption isotherms showed the typical hysteresis loop, which indicated that the existence of mesoporous structure. The pore size distribution shown in Fig. 3(D) also support the existence of mesoporous structure. The microencapsulated OMC/ZIF-8 materials showed the pore size at 10-20 nm. Otherwise, blank ZIF-8 materials showed the larger pore size at 20-30 nm. Combining with SEM results shown in Fig. 2, the mesoporous structure of OMC/ZIF-8 could be induced by the stack effect of the discrete ZIF-8 crystalline nanoparticles.

Based on these $\mathrm{N}_{2}$ sorption isotherms, the pore structure parameters have been calculated and listed in Table 1 . The specific surface area of $1131.9 \mathrm{~m}^{2} \mathrm{~g}^{-1}$ (BET method) and a pore volume of $0.53 \mathrm{~cm}^{3} \mathrm{~g}^{-1}$ were observed for the blank ZIF-8 materials. Moreover, according to $t$-plot method, the microporous inner surface area was about $1087.5 \mathrm{~m}^{2} \mathrm{~g}^{-1}$, which demonstrated the large inner porous surface area of ZIF-8 materials. On the other hand, OMC/ZIF-8 showed a decreased specific surface area of $953.9 \mathrm{~m}^{2} \mathrm{~g}^{-1}$ and a smaller pore volume

Table 1 Pore parameters of blank ZIF- 8 and microencapsulated OMC/ZIF-8

Samples

$S_{\text {Lang }}\left(\mathrm{m}^{2} \mathrm{~g}^{-1}\right)$

$S_{\mathrm{BET}}\left(\mathrm{m}^{2} \mathrm{~g}^{-1}\right)$

$S_{\text {ext }}\left(\mathrm{m}^{2} \mathrm{~g}^{-1}\right)$

$S_{\text {micro }}\left(\mathrm{m}^{2} \mathrm{~g}^{-1}\right)$

Pore volume $\left(\mathrm{cm}^{3} \mathrm{~g}^{-1}\right)$

Average pore width/nm

$\begin{array}{cc}\text { ZIF-8 } & \text { OMC/ZIF-8 } \\ 1531.2 & 1289.8 \\ 1131.9 & 953.9 \\ 44.4 & 19.4 \\ 1087.5 & 934.5 \\ 0.53 & 0.44 \\ 22.5 & 15.3\end{array}$


of $0.44 \mathrm{~cm}^{3} \mathrm{~g}^{-1}$. Moreover, the average pore width of OMC/ZIF-8 was $15.3 \mathrm{~nm}$. These results indicate that zeolitic imidazole frameworks has huge in-pore volume which could provide sufficient space for microencapsulation of organic UV filters.

For organic UV filters, the fast photochemical decomposition not only reduces the photo protection efficiency, but also produce phototoxic and photoallergenic degradation species. The photostability of free OMC and microencapsulated OMC in ZIF-8 has been studied and the results have been shown in Fig. 4(A) and (B). Comparing with free OMC, OMC/ZIF-8 has a greater UV absorbance between 250-350 $\mathrm{nm}$. The photoprotection performance improvement could be induced by the hydrophobic interaction between OMC and hydrophobic inner space of ZIF-8. On the other hand, the photostability of microencapsulated OMC/ZIF-8 has been improved comparing with free OMC. For free OMC, the UV absorbance has only remained $41 \%$ after $4 \mathrm{~h}$ irradiation. In comparison, microencapsulated OMC/ZIF-8 has retained 76\% of initial UV absorbance after $4 \mathrm{~h}$ irradiation. Totally, microencapsulation of OMC in ZIF-8 could not only enhance the photoprotection performance, but also improve the photostability of OMC UV filter.

The efficiency of UV filters could be estimated through measuring their UV absorption efficiency. The UV absorption efficiency of several samples of OMC in water, oil, ethanol and ZIF-8 has been determined and the corresponding results have been shown in Fig. 4(C). All the samples containing OMC had the same concentration of OMC at $0.01 \mathrm{~g} \mathrm{~L}^{-1}$. Comparing with OMC/water, OMC/ZIF-8 showed an 8-fold higher absorption.
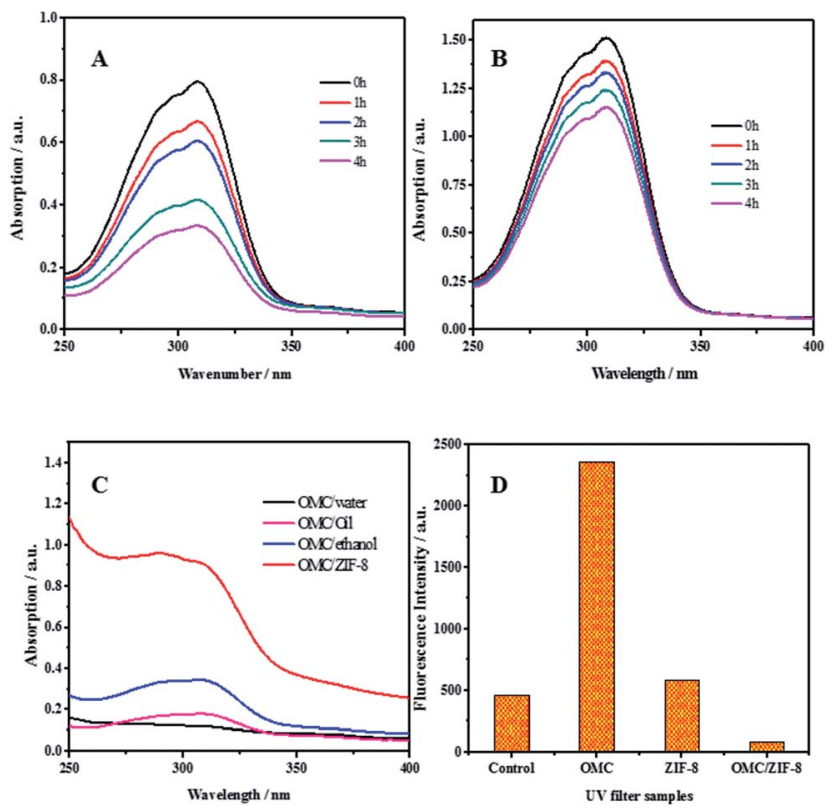

Fig. 4 UV protection effectiveness of free $O M C$ and microencapsulated OMC/ZIF-8. (A) UV absorbance variations of free OMC under UV irradiation. (B) UV absorbance variations of microencapsulated OMC in ZIF-8 under UV irradiation. (C) UV absorbance efficiency of OMC/water, OMC/oil, OMC/ethanol and OMC/ZIF-8 at OMC concentration of $0.01 \mathrm{~g} \mathrm{~L}^{-1}$ (D) ROS formation as measured by $\mathrm{DHR}$ fluorescence after UV irradiation. DHR was incubated with $\mathrm{OMC/}$ ZIF-8, blank ZIF-8, free OMC and PBS control.
With OMC/oil as the representative version of traditional sunscreen formulation, OMC/ZIF-8 showed a 5-fold higher absorption, which indicate that $\mathrm{OMC} / \mathrm{ZIF}-8$ could provide the higher UV photoprotective performance. The UV absorption efficiency of OMC/ethanol has also been determined, and the UV absorbance of OMC/ZIF-8 is about 3 times that of OMC/ ethanol. According to Saltzman's viewpoint, ${ }^{30}$ the UV absorption improvement is induced by improved solubility of organic UV filters in formulation. Moreover, Coudert et $a l^{\mathbf{4 0}}$ has reported that ZIF-8 had hydrophobic inner space, which could provide the more hydrophobic micro-environment for OMC. These results demonstrate that microencapsulated OMC in ZIF8 could provide much higher UV absorption efficiency comparing with traditional formulation.

Under UV irradiation, UV filter could produce toxic intermediates, such as ROS, which could induce oxidative pressure in cells, and destroy multiple cellular components, such as cell membrane, DNA and collagen protein. It has been reported that encapsulated UV filters in polymeric nanoparticles ${ }^{21}$ and biobased polysaccharide nanoparticles ${ }^{23}$ could improve the photostability and delay the photodegradation of UV filters. Therefore, we hypothesize that ZIF-8 could confine any ROS species generated from encapsulated OMC under UV irradiation into the nanoporous structure, and eliminate potential phototoxicity. In order to disclose the ROS species formation process, dihydrorhodamine (DHR) was used as ROS probe to detect ROS generated from OMC after UV irradiation. DHR was mixed with PBS control, free OMC, blank ZIF-8 and encapsulated OMC/ZIF-8, respectively. The fluorescence intensity was recorded and the results have been shown in Fig. 4(D). DHR in PBS solution could produce fluorescent emission because it absorbs UV between 380-320 $\mathrm{nm}$. In comparison, the fluorescence from free $\mathrm{OMC}$ is higher than the control, which demonstrates that more free ROS intermediates generated from the photoactivated OMC oxidized the DHR into fluorescent species. Meanwhile, the fluorescence production of ZIF- 8 could be induced by the UV absorbance originated from unsaturated $\mathrm{C}=\mathrm{N}$ structure in the zeolitic imidazole frameworks structure. In contrast, microencapsulated OMC/ZIF-8 shows lower fluorescent intensity, which could be ascribed to the confined ROS intermediates into ZIF-8 microporous structure, inhibiting the contact and reaction with DHR probe molecules.

In order to evaluate the stability of microencapsulated OMC in ZIF-8, the OMC release of microencapsulated OMC/ZIF-8 in artificial human sweat has been measured, and the corresponding results have been shown in Fig. 5. As we know, ZIF-8 is a kind of microporous metal organic framework material, which could provide high specific surface area and small pore size. The small pore size could provide high diffusion resistance for diffusion process. As shown in Fig. 5(A), OMC/ZIF-8 has released a few OMC molecules with diffusion time. Meanwhile, the release rate at $37{ }^{\circ} \mathrm{C}$ is higher than that at $32{ }^{\circ} \mathrm{C}$, which indicates that higher temperature could accelerate the molecule diffusion. After $24 \mathrm{~h}, \mathrm{OMC} / \mathrm{ZIF}-8$ released only $1.9 \%$ and $2.5 \%$ of the total OMC load at $32{ }^{\circ} \mathrm{C}$ and $37^{\circ} \mathrm{C}$. The OMC release amount of OMC/ZIF-8 is very low, which could be induced by confinement effect of regular microporous structure. These results 

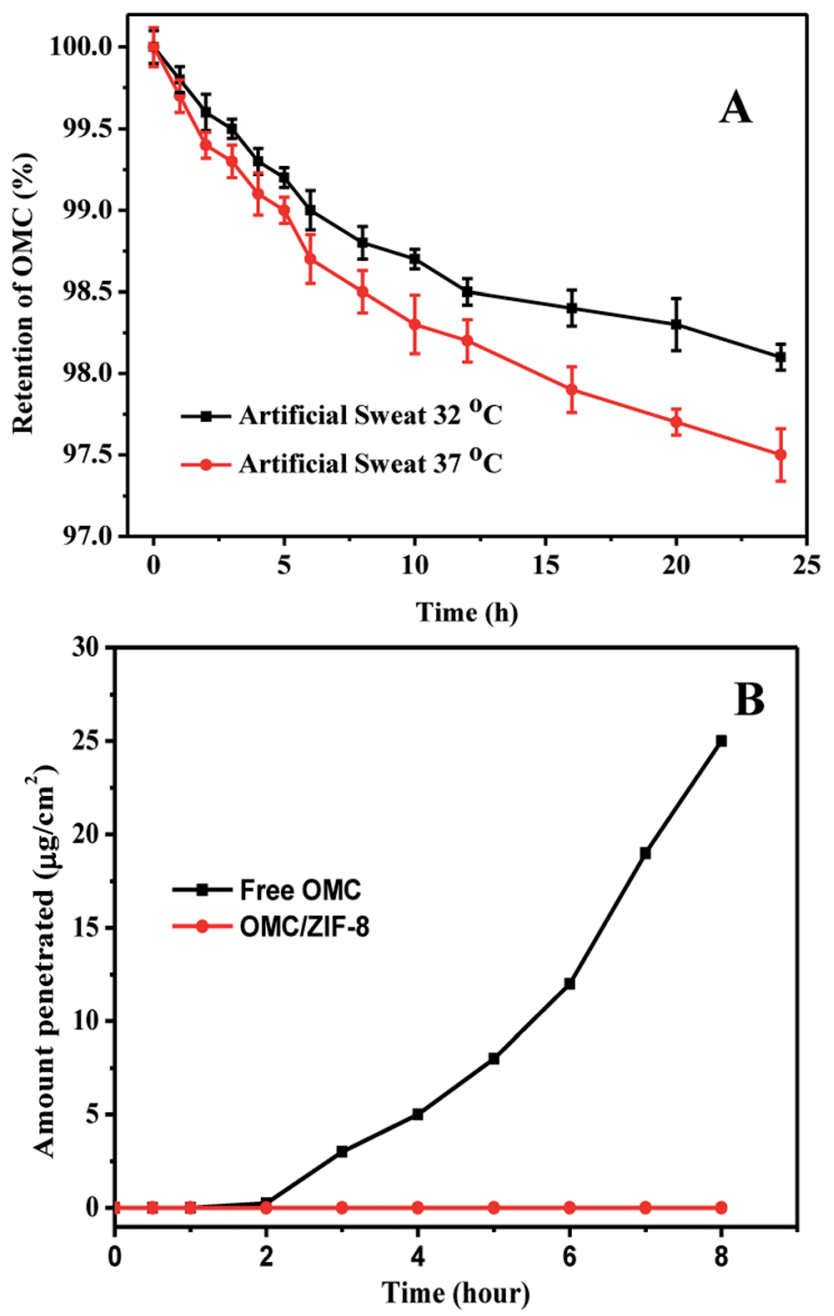

Fig. 5 Encapsulation stability and transdermal penetration of microencapsulated OMC/ZIF-8. (A) OMC retention within OMC/ZIF-8 in artificial human sweat at $32^{\circ} \mathrm{C}$ and $37^{\circ} \mathrm{C}$; (B) transdermal penetration of free and microencapsulated OMC in ZIF-8.

indicate that ZIF-8 could provide the high encapsulation stability for OMC UV filter, and decrease the direct contact risk of organic UV filter with skin.

In order to determine the transdermal diffusion of these microencapsulated OMC in vitro, Franz diffusion cell was used to analyze the effect of microencapsulation of OMC in ZIF-8 on transdermal penetration test. The corresponding results have been shown in Fig. 5(B). Free OMC could pass through human skin tissue into the receiver solution, and the drug flux was up to about $25 \mu \mathrm{g} \mathrm{cm}^{-2}$ at $8 \mathrm{~h}$. The transdermal penetration of free OMC could be induced by the hydrophobic interactions of OMC and skin tissues. In contrast, microencapsulated OMC/ZIF-8 samples were not detected in the receiver solution at any time. Although sweat pore is larger than the size of OMC/ZIF-8, OMC/ZIF-8 nanoparticles couldn't penetrate the skin through sweat pore, because the sweat gland is lined with sweatproducing cells, not open up into the body. Therefore, we speculate that the micro-encapsulated OMC in ZIF-8 samples remained on the surface of skin. Moreover, the microporous structure and small pore size could inhibit the leakage of OMC molecules, which could contribute to the zero penetration of OMC.

\section{Conclusions}

In conclusion, the microencapsulated UV filter such as OMC into nanoporous structure of ZIF-8 was demonstrated. The results showed that microencapsulated OMC could produce a 5fold greater UV spectral absorption than traditional OMC formulation due to nanoconfinement effect. Moreover, the obvious photostability improvement of OMC/ZIF-8 was demonstrated comparing with free OMC UV filter. More importantly, microencapsulated OMC into ZIF-8 could not only reduce the OMC release, but also confine the deleterious ROS intermediates originated from photo decomposition of OMC into the nanoporous structure of ZIF-8, which has prevented skin exposure to the UV filter molecules and the subsequent ROS intermediates. The UV filter/ZIF possess such advantages as improved photoprotective ability, photostability, and reduced risk of direct skin exposure, which would find broad application in safe and efficient sunscreen for UV protection.

\section{Conflicts of interest}

There are no conflicts to declare.

\section{Acknowledgements}

The authors are grateful for the financial support by the National Natural Science Foundation of China (No. 81771512, 21376120 and 81573072), and the Priority Academic Program Development (PAPD) of Jiangsu Higher Education Institutions.

\section{Notes and references}

1 J. L. Esbenshade, J. C. Cardoso and M. V. B. Zanoni, J. Photochem. Photobiol., A, 2010, 214, 257-263.

2 Y. Matsumura and H. N. Ananthaswamy, Toxicol. Appl. Pharmacol., 2004, 195, 298-308.

3 F. Aubin, Eur. J. Dermatol., 2003, 13, 515-523.

4 R. J. Witorsch and J. A. Thomas, Crit. Rev. Toxicol., 2010, 40, 1-30.

5 D. Dondi, A. Albini and N. Serpone, Photochem. Photobiol. Sci., 2006, 5, 835-843.

6 D. L. Giokas, A. Salvador and A. Chisvert, TrAC, Trends Anal. Chem., 2007, 26, 360-374.

7 M. D. Palm and M. N. O'Donoghue, Dermatol. Ther., 2007, 20, 360-376.

8 T. G. Smijs and S. Pavel, Nanotechnol., Sci. Appl., 2011, 4, 95112.

9 I. Fenoglio, J. Ponti, E. Alloa, M. Ghiazza, I. Corazzari, R. Capomaccio, D. Rembges, S. Oliaro-Bossoc and F. Rossi, Nanoscale, 2013, 5, 6567-6576.

10 G. A. Sotiriou, C. Watson, K. M. Murdaugh, T. H. Darrah, G. Pyrgiotakis, A. Elder, J. D. Brain and P. Demokritou, Environ. Sci.: Nano, 2014, 1, 144-153.

11 N. Shandilya and I. Capron, RSC Adv., 2017, 7, 20430-20439. 
12 M. Krause, A. Klit, M. Blomberg Jensen, T. Søeborg, H. Frederiksen, M. Schlumpf, W. Lichtensteiger, N. E. Skakkebaek and K. T. Drzewiecki, Int. J. Androl., 2012, 35, 424-436.

13 M. Schlumpf, K. Kypke, C. C. Vökt, M. Birchler, S. Durrer, O. Faass, C. Ehnes, M. Fuetsch, C. Gaille, M. Henseler, L. Hofkamp, K. Maerkel, S. Reolon, A. Zenker, B. Timms, J. A. F. Tresguerres and W. Lichtensteiger, Chimia, 2008, 62, 345-351.

14 M. Schlumpf, K. Kypke, M. Wittassek, J. Angerer, H. Mascher, D. Mascher, C. Vökt, M. Birchler and W. Lichtensteiger, Chemosphere, 2010, 81, 1171-1183.

15 C. G. J. Hayden, S. E. Cross, C. Anderson, N. A. Saunders and M. S. Roberts, Skin Pharmacol. Physiol., 2005, 18, 170-174.

16 E. Gilbert, F. Pirot, L. Roussel, F. Falson and K. Padois, Int. J. Cosmet. Sci., 2013, 35, 208-219.

17 M. Schlumpf, B. Cotton, M. Conscience, V. Haller, B. Steinmann and W. Lichtensteiger, Environ. Health Perspect., 2001, 109, 239-244.

18 M. Axelstad, J. Boberg, K. S. Hougaard, S. Christiansen, P. R. Jacobsen, K. R. Mandrup, C. Nellemann, S. P. Lund and U. Hass, Toxicol. Appl. Pharmacol., 2011, 250, 278-290.

19 M. Schlumpf, P. Schmid, S. Durrer, M. Conscience, K. Maerkel, M. Henseler, M. Gruetter, I. Herzog, S. Reolon, R. Ceccatelli, O. Faass, E. Stutz, H. Jarry, W. Wuttke and W. Lichtensteiger, Toxicology, 2004, 205, 113-122.

20 N. Duale, A.-K. Olsen, T. Christensen, S. T. Butt and G. Brunborg, Toxicol. Sci., 2010, 114, 272-284.

21 Y. Deng, A. Ediriwickrema, F. Yang, J. Lewis, M. Girardi and W. M. A. Saltzman, Nat. Mater., 2015, 14, 1278-1285.

22 S. H. Tolbert, P. D. McFadden and D. A. Loy, ACS Appl. Mater. Interfaces, 2016, 8, 3160-3174.

23 D. R. Hayden, A. Imhof and K. P. Velikov, ACS Appl. Mater. Interfaces, 2016, 8, 32655-32660.

24 M. M. Jiménez, J. Pelletier, M. F. Bobin and M. C. Martini, Int. J. Pharm., 2004, 272, 45-55.
25 P. Perugini, S. Simeoni, S. Scalia, I. Genta, T. Modena, B. Conti and F. Pavanetto, Int. J. Pharm., 2002, 246, 37-45.

26 Y. Bai, Y. Dou, L.-H. Xie, W. Rutledge, J.-R. Li and H.-C. Zhou, Chem. Soc. Rev., 2016, 45, 2327-2367.

27 O. M. Yaghi, J. Am. Chem. Soc., 2016, 138, 15507-15509.

28 K. S. Park, Z. Ni, A. P. Côté, J. Y. Choi, R. Huang, F. J. UribeRomo, H. K. Chae, M. O'Keeffe and O. M. Yaghi, Proc. Natl. Acad. Sci. U. S. A., 2006, 103, 10186-10191.

29 X. C. Huang, Y. Y. Lin, J. P. Zhang and X. M. Chen, Angew. Chem., Int. Ed., 2006, 45, 1557-1559.

30 J.-P. Zhang, A.-X. Zhu and X. M. Chen, Chem. Commun., 2012, 48, 11395-11397.

31 J.-W. Ye, H.-L. Zhou, S.-Y. Liu, X.-N. Cheng, R.-B. Lin, X.-L. Qi, J.-P. Zhang and X.-M. Chen, Chem. Mater., 2015, 27, 8255-8260.

32 J.-W. Ye, X. Zhou, Y. Wang, R. K. Huang, H. L. Zhou, X. N. Cheng, Y. Ma and J.-P. Zhang, Sci. China Mater., 2018, 61, 424-428.

33 B. Wang, X.-L. Lv, D. Feng, L.-H. Xie, J. Zhang, M. Li, Y. Xie, J.-R. Li and H.-C. Zhou, J. Am. Chem. Soc., 2016, 138, 62046216.

34 S. Liu, L. Wang, J. Tian, Y. Luo, G. Chang, A. M. Asiri, A. O. AlYoubi and X. Sun, ChemPlusChem, 2012, 77, 23-26.

35 H. Li, X. Feng, Y. Guo, D. Chen, R. Li, X. Ren, X. Jiang, Y. Dong and B. Wang, Sci. Rep., 2014, 4, 4366-4370.

36 J. Park, Q. Jiang, D. Feng, L. Mao and H.-C. Zhou, J. Am. Chem. Soc., 2016, 138, 3518-3525.

37 H. Zheng, Y. Zhang, L. Liu, W. Wan, P. Guo, A. M. Nyström and X. Zou, J. Am. Chem. Soc., 2016, 138, 962-968.

38 J. Cravillon, S. Münzer, S. Lohmeier, A. Feldhoff, K. Huber and M. Wiebcke, Chem. Mater., 2009, 21, 1410-1412.

39 Y. Hu, H. Kazemian, S. Rohani, Y. Huang and Y. Song, Chem. Commun., 2011, 47, 12694-12696.

40 A. U. Ortiz, A. P. Freitas, A. Boutin, A. H. Fuchs and F. X. Coudert, Phys. Chem. Chem. Phys., 2014, 16, 9940-9949. 